\title{
Detección de trastornos de la conducta alimentaria en una unidad de alcoholismo.
}

Detection of eating behavior disorders in an alcoholism unit.

\author{
María José Muñoz Algar a, Patricia Bernal García ${ }^{\text {b }}$, Félix Poyo Calvo ${ }^{\text {c }}$. \\ ${ }^{a, b y c}$ Psiquiatra. ${ }^{a}$ Instituto Psiquiátrico Montreal. El Plantío, Majadahonda. Madrid, España. ${ }^{b}$ Centro de Salud \\ Mental Puente de Vallecas. Madrid, España. ${ }^{c}$ Jefe coordinador de la sección de adicciones. Hospital Doctor \\ Rodríguez Lafora. Madrid, España.
}

Correspondencia: María José Muñoz_Algar (maria_jose_m@msn.com)

Recibido: 03/09/2014; aceptado: 05/05/2015

RESUMEN: Introducción: La coexistencia de trastornos alimentarios (TCA) y de sustancias ha sido el foco de investigaciones clínicas desde hace varias décadas con amplia y contradictoria literatura. El objetivo del estudio es detectar la presencia de trastornos alimentarios en una muestra de pacientes alcohólicos. Material y métodos: Estudio prospectivo de 340 pacientes ingresados en la Unidad de Deshabituación Alcohólica (U.D.A.) del Hospital Dr Rodríguez Lafora, realizado a lo largo de 18 meses. En los casos en los que se diagnosticó un trastorno de la conducta alimentaria, en 30 mujeres (25\% de la muestra femenina) y ningún hombre, se analizaron las características demográficas, psicosociales y psicopatológicas. Además se estudió el trastorno alimentario y se realizaron varios test psicométricos específicos. Resultados: Encontramos una elevada comorbilidad psiquiátrica en 3/4 partes de la muestra (patología ansiosa y depresiva), elevados antecedentes familiares de alcoholismo y malos tratos, y elevadas cifras de policonsumo de tóxicos. Por otro lado, más de la mitad de la muestra reconoce haber utilizado el alcohol con fin anorexígeno, lo que nos sugiere que al alcohol cumpliría una función dentro del trastorno alimentario. Conclusiones: Dado que hemos encontrado que un número importante de pacientes que inicialmente padece trastorno alimentario desarrollan alcoholismo en el curso de la enfermedad, consideramos que en estas pacientes debería realizarse de forma sistemática una evaluación del consumo de alcohol y otros tóxicos. La detección temprana de esta comorbilidad facilitaría un abordaje e intervención terapéutica más específicos.

PALABRAS CLAVE: Alcoholismo, trastorno alimentario, detección, comorbilidad, anorexígeno.
ABSTRACT: Introduction: The coexistence of eating behaviour disorders and substance abuse has been in the focus of clinical research for several decades with large and contradictory literature results. The aim of this study is to detect eating behaviour disorders in a sample of alcoholic patients. Material and methods: Prospective study of 340 patients admitted in an alcohol dishabituation unit (A.D.U.) from Dr Rodríguez Lafora Hospital during a period of 18 months. In cases diagnosed with an eating disorder, 30 women ( $25 \%$ of the women sample) and no men, we analyzed the demographic, psychosocial and psychopathological characteristics. Furthermore, we studied the eating disorder and we realized several specific psychometric tests. Results: We found a high psychiatric comorbidity in three quarters of the sample (anxious and depressive pathology), high rates of alcohol abuse in the family; and high rates of drug misuse. On the other hand, over half of the sample recognized to use alcohol as an anorectic substance, suggesting that alcohol had a function within eating disorders. Conclusions: Since we have found a significant number of patients that initially were suffering from eating disorders and they developed alcoholism in the course of the disease, we consider in this kind of patient that is necessary to make a systematic assessment of alcohol and other substances. Early detection of this comorbidity is useful for achieve a more specific approach and therapeutic intervention.

KEY WORDS: Alcoholism, eating behaviour, detection, comorbidity, anorexigenic.

Agradecimiento: Los autores agradecen la colaboración de C. Salanova Otero en la recogida de datos.

Conflicto de intereses: Los autores declaran que no tienen conflicto de intereses en este estudio. 


\section{Introducción}

La coexistencia de trastornos alimentarios (TCA) y de sustancias ha sido el foco de investigaciones clínicas desde hace varias décadas (1-2), con amplia y contradictoria literatura (3). La evidencia teórica y empírica apoya la relación entre los TCA y en concreto, con el alcoholismo (4-7).

Sin embargo, aún quedan muchos puntos que aclarar en dicha comorbilidad, como el de la influencia de un trastorno sobre el otro (8). Por otro lado, encontramos que la mayoría de estudios provienen de centros especializados en el tratamiento de TCA (4), con muestras de niñas y adolescentes en edad escolar (9), universidades y colegios mayores $(6,10-12)$ y deportistas $(8)$. Pero son muy escasos los trabajos que abordan este tema desde el punto de vista de unidades de tratamiento del alcoholismo (13). Cabe destacar que el abordaje sea realizado mayoritariamente en población femenina, ya que la prevalencia encontrada en hombres es baja (14).

Existe una elevada prevalencia de abuso de sustancias entre pacientes con TCA (15-16). Los distintos trastornos de la conducta alimentaria presentan diferentes tasas de comorbilidad con uso y abuso de sustancias, con resultados que sugieren una sobrerrepresentación (17). La bulimia nerviosa es la enfermedad en la que se han descrito mayor número de trastornos por abuso de sustancias $(6,18$ $20)$, siendo la prevalencia en torno al 2,6-60\% $(3,7,21)$, mayor que en anoréxicas purgativas y a su vez que en las restrictivas, con una prevalencia mayor del $23 \%$ en anorexia nerviosa (siendo el alcohol, como ya hemos comentado, y los estimulantes las más utilizadas $(19,22-23))$. Por otro lado, hemos encontrado que el 35-40\% de alcohólicos tienen historia de TCA (24). Estos índices, ya sea desde el punto de vista del trastorno alimentario o de la dependencia alcohólica, exceden a la población general $(4,25)$.

El objetivo de nuestro estudio es detectar la presencia de TCA en una muestra de mujeres que realizan tratamiento para su dependencia alcohólica, y evaluar la relación existente entre ambos.

\section{Método}

Estudio prospectivo de pacientes ingresados en la Unidad de Deshabituación Alcohólica (U.D.A.) del Hospital Dr Rodríguez Lafora realizado a lo largo de 18 meses. Durante este período, 120 mujeres y 220 hombres fueron ingresados $(\mathrm{N}=340)$. 
ORIGINALES BREVES

\section{Procedimiento}

Los 340 pacientes fueron evaluados consecutivamente tras ingresar en la U.D.A. Toda la muestra cumplía criterios de dependencia alcohólica según criterios DSM-IV y se encontraban en abstinencia de alcohol y otros tóxicos durante su ingreso. Se realizó una entrevista de evaluación y un test de screening (Body Shape Questionnaire, BSQ) a toda la muestra. En los casos en los que se diagnosticó un Trastorno de la Conducta Alimentaria (TCA) $(n=30)$, se analizaron las características demográficas, psicosociales y psicopatológicas de la muestra, se estudió el trastorno alimentario y se realizaron varios test psicométricos específicos.

\section{Instrumentos}

Relacionados con la esfera alimentaria.

Body Shape Questionnaire (BSQ) (26). Cuestionario autoaplicado que consta de 34 ítems que se puntúan en una escala de Likert de 1 a 6 . Se deriva en cuatro subescalas: insatisfacción corporal, miedo a engordar, baja estima por la apariencia y deseo de perder peso. El punto de corte para la puntuación total se ha establecido en 105.

\section{Relacionados con las dimensiones de la personalidad.}

Escala de Búsqueda de Sensaciones (SSS) (27) (validada por Pérez y Torrubia (28)). La escala está compuesta por 40 ítems, que se contestan de forma autoaplicada con la indicación de verdadero o falso. Cada respuesta afirmativa equivale a un punto y cada respuesta negativa a 0 . No existen puntos de corte propuestos. Cuanto mayor es la puntuación, mayor es la presencia del rasgo. La escala recoge cuatro dimensiones, que son la búsqueda de emociones, la búsqueda de excitación, la desinhibición y la susceptibilidad al aburrimiento.

Escala de Impulsividad (29) (versión española de Luengo, Carrillo de la Peña y Otero (30)). Se trata de una escala autoaplicada que consta de 33 ítems, con 5 opciones de respuesta, que puntúan de 0 a 4 (casi nunca/nunca, algunas veces, a veces sí/a veces no, bastantes veces, casi siempre/siempre). Según Barratt, se pueden precisar 3 tipos de impulsividad: la impulsividad motora, la impulsividad cognitiva y la improvisación/ausencia de planificación. La puntuación total se obtiene sumando las puntuaciones obtenidas. No existe un punto de corte propuesto. Desde la perspectiva clínica, lo que va a tener mayor importancia es el valor cuantitativo de la puntuación total.

Cuestionario de Ansiedad-Riesgo (STAI-R). Es una parte del cuestionario de 
ansiedad estado-rasgo (31), versión española de TEA (32). Se valora la ansiedadrasgo como una predisposición ansiosa estable a percibir las situaciones como amenazantes. Consta de 20 ítems, que miden la ansiedad como rasgo, según una escala de tipo Likert que va de 0 a 3 (nada, algo, bastante, mucho). La puntuación total oscilará entre 0 y 60 . El punto de corte para la población masculina es de 25 y para la población femenina de 32 .

\section{Relacionadas con las características psicopatológicas.}

Inventario de Depresión de Beck (BDI). Es un autoinforme que evalúa la presencia e intensidad de sintomatología depresiva y está validado para la población española (33). La versión utilizada consta de 21 apartados con diferentes opciones de respuesta (entre 4 y 8), cada una de las cuales tiene un valor entre 0 y 3 , en donde 0 representa la ausencia de sintomatología y 3 la máxima gravedad. Se toman en consideración las siguientes puntuaciones: 0-9, ausencia de depresión; 10-18, sintomatología depresiva leve; 19-29 sintomatología depresiva moderada; e igual o mayor que 30 , sintomatología depresiva grave.

\section{Resultados}

En el total de 340 pacientes, no hemos encontrado ningún TCA entre los 220 hombres. En el caso de las 120 mujeres, detectamos 30 que cumplían criterios de TCA ( $25 \%$ de la muestra).

\section{Caracteristicas de la muestra}

Análisis sociodemográfico. (Tabla 1) Las 30 pacientes diagnosticadas de TCA y dependencia alcohólica tienen una edad media de 40,4 años (rango de 2763 años de edad), siendo toda la muestra de raza caucásica. Mayoritariamente son mujeres solteras que conviven con la familia de origen, tienen un nivel educacional medio y están desempleadas.

Análisis del consumo de tóxicos y antecedentes psiquiátricos. (Tabla 2) El 96,6\% de la muestra consume otros tóxicos (excluyendo el alcohol): siendo los más consumidos el tabaco $(86,2 \%)$, cocaína $(44,8 \%$ ) y benzodiacepinas, en el $38 \%$ de la muestra. Por otro lado, destaca el consumo de otras sustancias como bebidas estimulantes carbonatadas en el $64,7 \%$ y café en un $41,2 \%$. Respecto a sus antecedentes, el 76,6\% de la muestra presenta comorbilidad psiquiátrica: 70\% trastornos ansiosodepresivos y $30 \%$ trastornos de personalidad; además el $60 \%$ presentan antecedentes de intentos autolíticos previos. El 46,6\% de la muestra tiene antecedentes familiares psiquiátricos y el 73,3\% presentan antecedentes familiares de alcoholismo en familiares de primer grado. Encontramos antecedentes de malos tratos físicos en 43,3\%, psicológicos en el $46,6 \%$ y abusos sexuales en $36,6 \%$ de la muestra. 


\section{ORIGINALES BREVES}

Tabla 1

Análisis sociodemográfico

\begin{tabular}{|c|c|}
\hline & $N$ \\
\hline \multicolumn{2}{|l|}{ Edad } \\
\hline Media $=40,4$ años & \\
\hline Rango $=27-63$ años & \\
\hline \multicolumn{2}{|l|}{ Raza } \\
\hline Caucásica & 30 \\
\hline Afroamericana, hispánica, otras. & 0 \\
\hline \multicolumn{2}{|l|}{ Estado civil } \\
\hline Soltera & 15 \\
\hline Casada & 7 \\
\hline Divorciada/Separada & 8 \\
\hline Viuda & 0 \\
\hline \multicolumn{2}{|l|}{ Convivencia: } \\
\hline Sola & 6 \\
\hline Familia de origen & 13 \\
\hline Familia propia & 11 \\
\hline \multicolumn{2}{|l|}{ Nivel educacional: } \\
\hline Primarios & 5 \\
\hline Medios & 16 \\
\hline Universitarios & 9 \\
\hline \multicolumn{2}{|l|}{ Nivel laboral } \\
\hline En activo & 9 \\
\hline Desempleado & 17 \\
\hline Baja laboral & 2 \\
\hline Ama de casa & 2 \\
\hline \multicolumn{2}{|l|}{ Nivel sociocultural } \\
\hline Bajo & 2 \\
\hline Medio & 24 \\
\hline Alto & 4 \\
\hline Problemas sociales: & 13 \\
\hline Inadaptación & $1 / 13$ \\
\hline Económicos & $8 / 13$ \\
\hline Laborales & $10 / 13$ \\
\hline
\end{tabular}

Tabla 2

Análisis del consumo de tóxicos y antecedentes psiquiátricos

\begin{tabular}{cr}
\hline & $N$ \\
\hline Consumo activo de tóxicos (excluyendo el alcohol) & 29 \\
Tabaco & $25 / 29$ \\
Cocaína & $13 / 29$ \\
Cannabis & $9 / 29$ \\
Anfetaminas & $7 / 29$ \\
Drogas de diseño & $4 / 29$ \\
Heroína & $0 / 29$ \\
Benzodiacepinas & $11 / 29$ \\
Otras adicciones & 17 \\
Bebidas estimulantes carbonatadas & $11 / 17$ \\
Red bull & $3 / 17$ \\
Café & $7 / 17$
\end{tabular}

Antecedentes psiquiátricos previos (excluyendo comorbilidad)

23

Depresión, ansiedad 16/23

T. Personalidad $7 / 23$

Antecedentes familiares de primer grado Psiquiátricos Alcoholismo

Suicidalidad Intentos autolíticos $\quad 18$ Autolesiones 10

Antedentes de maltrato Malos tratos físicos $\quad 13$

Abusos sexuales

Análisis del propio TCA (Tabla 3). El índice de masa corporal promedio de la muestra es 23,57 , encontrando que $2 / 3$ partes de la muestra se encuentran en normopeso y $1 / 3$ en sobrepeso. El 73,3\% verbalizó que desearía tener un peso inferior al que posee en la actualidad.

Cuando nos centramos en el análisis del TCA comórbido, el 63,3\% inician el trastorno alimentario en la adolescencia. El diagnóstico de TCA inicial fue: AN purgativa y $\mathrm{BN}$ en el $33,3 \%$ respectivamente, AN restrictiva en el $26,6 \%$ y TCA no especificado en el 6,6\%. Analizando la cronología, el 86,6\% de las pacientes desarrollan el trastorno alimentario previo al inicio de la dependencia alcohólica, y el $100 \%$ de la muestra presentan en la actualidad un TCA activo, con una adecua- 
Tabla 3

Análisis del propio TCA

\begin{tabular}{lr}
\multicolumn{2}{c}{ Análisis del propio TCA } \\
\hline \multicolumn{1}{c}{$N$} \\
\hline Indice de masa corporal \\
Media & 23,57 \\
Infrapeso & 1 \\
Normopeso & 19 \\
Sobrepeso & 10
\end{tabular}

Peso deseado respecto al actual

$\begin{array}{lr}\text { Inferior } & 22 \\ \text { Igual } & 5 \\ \text { Superior } & 3\end{array}$

Edad de inicio del TCA

Adolescencia $\quad 19$

Adultez 11

Cronología del TCA

TCA previo a la dependencia de alcohol 26

TCA posterior a la dependencia de alcohol 4 Evolución del TCA: diagnóstico presente en la actualidad $\quad 30$

Diagnóstico TCA inicial

$\begin{array}{lr}\text { AN restrictiva } & 8 \\ \text { AN purgativa } & 10 \\ \text { B. Nerviosa } & 10 \\ \text { TCA no especificado } & 2\end{array}$

Conductas realizadas para perder peso (en la actualidad)

$\begin{array}{lr}\text { Vómitos } & 9 \\ \text { Laxantes/diuréticos } & 4 \\ \text { Dieta } & 30 \\ \text { Ayuno } & 9 \\ \text { Ejercicio excesivo } & 6 \\ \text { emas somáticos derivados del TCA } & 7 \\ \text { d ante la enfermedad } & \\ \text { Negación } & 2 \\ \text { Minimización/ambivalencia } & 2 \\ \text { Aceptación } & 26\end{array}$

Existen período de abstinencia del alcohol $>6$ meses 20

Evolución del TCA durante la abstinencia de alco-

hol (en la submuestra descrita)

Permanecen mismo síntomas $\quad 7 / 20$

Empeora el TCA 2/20

Mejora el TCA 11/20

Efectos buscados con el alcohol

Desinhibidor, euforizante 24

Sedante, relajante 22

Anorexígeno 17

Pacientes que encuentran relación entre el TCA y el

$\begin{array}{ll}\text { consumo de alcohol 23/30 } & \end{array}$ 
da conciencia de enfermedad en el $86,6 \%$ de ellas. Las conductas realizadas para perder peso son: la dieta en el $100 \%$, ayuno y vómitos en un $30 \%$ respectivamente, ejercicio excesivo en el 20\% y laxantes y/o diuréticos en solo un 13,3\%.

Encontramos que un $66,7 \%$ de la muestra reconoce haber tenido un período de abstinencia superior a 6 meses, durante los cuales, el 55\% mejora el trastorno alimentario, en el 35\% permanecen los mismos síntomas y el 10\% empeora el trastorno alimentario.

Entre los efectos más buscados con el consumo del alcohol: $80 \%$ desinhibidor, $73,3 \%$ relajante y el 56,6\% anorexígeno. A su vez, el 76,6\% refiere encontrar una relación entre el consumo de alcohol y su trastorno alimentario.

\section{Pruebas psicométricas (Tabla 4)}

Ansiedad (STAI-R). La muestra de pacientes comórbidas presenta niveles de ansiedad en el centil 60 respecto a la población general.

Impulsividad (BIS-11). Encontramos elevadas cifras de impulsividad a expensas de mayor improvisación y ausencia de planificación $(20,9 \pm 7,94)$.

Búsqueda de sensaciones (SSS). La puntuación obtenida por la muestra a estudio es de 18,16 $\pm 5,3$ con mayores puntuaciones en la subescala de búsqueda de excitación.

Depresión (BDI). El 36,6\% de la muestra puntúa en depresión leve y el 23,4\% en depresión moderada. Solo el $40 \%$ de las pacientes presenta ausencia de depresión.

Tabla 4

Pruebas psicométricas

\begin{tabular}{|c|c|}
\hline & $X(D T)$ \\
\hline $\begin{array}{l}\text { Ansiedad (STAI-R) } \\
\text { (Rango:0-60)(corte en 32) }\end{array}$ & $27,13(4,75)$ \\
\hline $\begin{array}{ll}\text { Impulsividad (BIS-11) } \\
\text { (Rango: } & 0-132 \text { ) } \\
- & \text { Impulsividad motora } \\
- & \text { Impulsividad cognitiva } \\
\text { - } & \text { Improvisación y ausencia de planificación. }\end{array}$ & $\begin{array}{c}60,6(19,4) \\
21,33(6,44) \\
17,23(6,61) \\
20,9(7,94)\end{array}$ \\
\hline 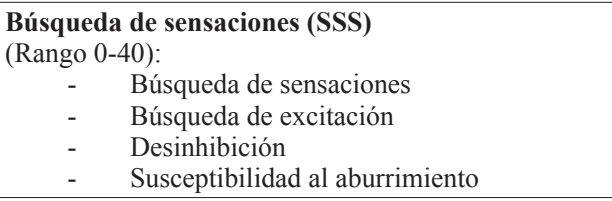 & $\begin{array}{l}18,16(5,3) \\
4,5(2,82) \\
5,8(1,88) \\
4,5(2,25) \\
3,36(1,88)\end{array}$ \\
\hline & $N(\%)$ \\
\hline $\begin{array}{l}\text { Depresión (BDI) } \\
\text { X }(\mathrm{DT})=12,56(7,72) \\
\text { Rangos de puntuación: }\end{array}$ & \\
\hline $\begin{array}{ll}\text { - } & \text { 0-9 ausencia de depresión } \\
\text { - } & \text { 10-18 depresión leve } \\
\text { - } & \text { 19-29 depresión moderada } \\
\text { - } & \text { 30-63 depresión grave }\end{array}$ & $\begin{array}{c}12(40 \%) \\
11(36,6 \%) \\
7(23,4 \%) \\
0\end{array}$ \\
\hline
\end{tabular}

$\mathrm{X}=$ Media, DT=Desviación Típica, STAI-R=Inventario de ansiedad-rasgo, BIS=Escala de impulsividad de Barratt, $\mathrm{SSS}=$ Escala de Búsqueda de Sensaciones de Zuckerman, BDI: Escala de depresión de Beck. 


\section{Discusión}

Las personas con trastornos alimentarios frecuentemente presentan comorbilidad psiquiátrica y psicosocial que afectan a todos los ámbitos de su vida (34). En nuestro estudio hemos encontrado resultados en la misma línea de las investigaciones publicadas previamente, con elevada prevalencia de comorbilidad psiquiátrica (aproximadamente $3 / 4$ de la muestra), encontrándose un $70 \%$ en tratamiento por trastornos ansioso-depresivos asociados. Esto queda reflejado en elevadas tasas de depresión en nuestra muestra (60\% con cifras en rango leve-moderado en el BDI) y niveles de ansiedad-rasgo con puntuación centil 60 respecto a población general. Por otro lado, la elevada prevalencia de antecedentes familiares de alcoholismo $(73,3 \%)$ podría sugerir que nos encontramos con un subgrupo de pacientes especialmente vulnerable al desarrollo de alcoholismo a lo largo de la vida (35). Autores como Harrel y cols. (36) proponen que la historia familiar de alcoholismo está relacionada con un consumo problemático de alcohol, pero sin embargo, encontraron limitaciones sobre cómo se relacionaba éste y otros cofactores en mujeres. Por otra parte, encontramos elevados antecedentes de malos tratos, en torno al $36,6-43,3 \%$, lo que podría indicar que nos encontramos ante pacientes con marcada vulnerabilidad y que podrían presentar cierto grado de indefensión.

Es destacable que nuestra muestra presenta elevadas cifras de policonsumo de tóxicos; en torno al $97 \%$ de las pacientes consumen al menos dos de ellos, siendo los más prevalentes aquellos de fácil acceso por ser "drogas legales". Las bebidas estimulantes (consumidas en cantidad variable), son otras de las sustancias que consume la mitad de la muestra, en concreto aquellas que poseen cafeína en su composición. Higuchi y cols. (14) hablan de la posible existencia de una "personalidad adictiva". Algunos especulan que los individuos que desarrollan adicción a una sustancia, desarrollan patrones psicológicos y de comportamiento que les hacen vulnerables para desarrollar adicción a otras sustancias. Grilo (17), por su parte, establece como factor común en ambas patologías el déficit en la regulación del control de impulsos y el afecto. Nuestra muestra, por otra parte, confirma esta afirmación presentando elevadas cifras de impulsividad.

Si nos centramos en la comorbilidad del TCA y la dependencia alcohólica, el trastorno alimentario aparece previo al establecimiento de la dependencia alcohólica en un $86,6 \%$ de las pacientes, mayoritariamente en la adolescencia, con mayor frecuencia de conductas de atracones y purgativas al inicio del trastorno (con diagnóstico de $\mathrm{BN}$ y AN purgativa en $2 / 3$ de la muestra) y la evolución de dicha comorbilidad con ambos trastornos presentes en el momento actual. Sin embargo, encontramos que todas ellas realizan dieta en la actualidad, con un descenso de las conductas purgativas y un aumento de las conductas restrictivas para mantener el peso en comparación a las realizadas cuando se inició el TCA. Además, más de la 
mitad de la muestra reconoce haber utilizado el alcohol con fin anorexígeno, lo que nos sugiere que el alcohol cumpliría una función dentro del trastorno alimentario, algo que en torno al $77 \%$ de la muestra verbaliza a lo largo de las entrevistas realizadas. Sin embargo, poco se ha escrito sobre la influencia de uno sobre otro (37). En aquellas pacientes que lograron mantener la abstinencia de alcohol durante un período de al menos 6 meses, en el 55\% de ellas mejoró el TCA y tan solo empeoró en el $10 \%$. Esto apoya nuestra hipótesis de que el alcohol influye negativamente en la evolución del trastorno alimentario comórbido.

Por último lugar, hemos encontrado limitaciones referidas a los participantes del estudio. Aunque analizamos una muestra amplia, encontramos un sesgo de selección al analizar pacientes ingresadas en un recurso de tercer nivel, altamente especializado en el tratamiento de pacientes con problemas de alcoholismo. Por otro lado, pensamos que la proporción de mujeres encontrada sigue siendo excesivamente baja respecto a la prevalencia real de alcoholismo femenino en la población general. Todo ello podría implicar que las pacientes que acuden a dicho recurso sean una parte de población más grave y exista el riesgo de no reflejar la población general. Por este motivo, debemos ser prudentes a la hora de generalizar los resultados.

\section{Conclusiones}

La coexistencia de ambos desórdenes posee implicaciones clínicas, terapéuticas y pronósticas (38).

Se recomienda realizar un screening de sustancias de abuso en pacientes con TCA de forma rutinaria (23). Un número importante de pacientes que inicialmente tienen TCA desarrollan alcoholismo en el curso de la enfermedad. De hecho, varios estudios identifican la impulsividad como factor pronóstico negativo a largo plazo (37). Por tanto, pensamos que el abordaje precoz del riesgo de dependencia alcohólica en pacientes diagnosticadas de TCA disminuiría la comorbilidad de ambos trastornos; disminuiría el riesgo de padecer trastorno por dependencia de alcohol y, como se ha evidenciado en el estudio, mejoraría asimismo el TCA. Esto supondría un evidente beneficio clínico para el paciente y una disminución de costes sociosanitarios.

Recomendamos ampliar las investigaciones, ya que mejorar la comprensión de cómo se influencian mutuamente ambas patologías nos permitiría entender la etiología y la prevención (39); todo ello para ofrecer un abordaje adecuado y una intervención terapéutica específica hacia este tipo de pacientes. 


\section{BIBLIOGRAFÍA}

(1) Westermeyer J and Specker S. "Social Resources and social Function in Comorbid Eating and Substance Disorder: A matched-Pairs Study". Am J Addict 1999; 8: 332-336.

(2) Jones A, Cheshire N and Moorhouse H. "Anorexia nervosa, bulimia and alcoholismassociation of eating disorder and alcohol". 1985. J. Psychiatry Res. Vol 19. No 2/3. Pp: 377-380.

(3) Bailly D. "Recherche épidémiologique, troubles du comportement alimentaire et conduites de dépendance”. L’Encéphale, 1993; XIX: 285-92.

(4) Goldbloom DS, Naranjo CA, Bremner KE \& Hicks LK. "Eating disorders and alcohol abuse in women". Br J Addict. 1992 Jun; 87(6):913-9.

(5) Schuckit MA, Tipp JE, Anthenelli RM, Bucholz KK, Hesselbrock VM \& Nurnberger JI Jr. Anorexia nervosa and bulimia nervosa in alcohol-dependent men and women and their relatives. Am J Psychiatry 1996. 153 (1): 74-82.

(6) Raeburn S.D. The Handbook of Addiction Treatment for Women: Theory and Practice. Chapter 6: Women and eating disorders. Chap. 6, pp. 127-153. Straussner, S. L. A. and Brown, S., eds. 2002, Jossey-Bass.

(7) Benjamin L \& Wulfert E. "Dispositional correlates of addictive behaviors in college women: Binge eating and heavy drinking". Eating Behaviors 6 (2005) 197-209.

(8) Gutgesell M.E. \& Timmerman M.G. "Alcohol use and behaviors in women long-distance race participants reporting a history of bulimia and/or anorexia nervosa". J Sports Med Phys Fitness. 1998. Vol 38. No 2. PP: 142-8.

(9) Krahn D D, Kurth C L, Gomberg E \& Drewnowski, A. "Pathological dieting and alcohol use in college women - a continuum of behaviors". Eating Behaviors 6 (2005) 43-52.

(10) Loxton NJ and Dawe S. Alcohol abuse and dysfunctional eating in adolescent girls: the influence of individual differences in sensitivity to reward and punishment. Int J Eat Disord. 2001 May; 29(4):455-462.

(11) Lundholm JK. "Alcohol use among university females: relationship to eating disordered behavior”. Addictive Behaviors, 1989. Vol 14. pp:181-185.

(12) Striegel-Moore R.H. "Problem drinking and symptoms of disordered eating in female high school students". 1993. International Journal of Eating Disorders, Vol 14. No 4: 417-425.

(13) Peveler R. \& Fairburn C. "Eating disorders in women who abuse alcohol". British Journal of Addiction". 1990. 85: 1633-1638.

(14) Higuchi S, Suzuki K, Yamada K, Parrish K and Kono H. "Alcoholics with eating disorders: Prevalence and Clinical course. A study from Japan”. British Journal of Psychiatry (1993). 162: 403-406.

(15) Wiseman CV, Sunday SR, Halligan P, Korn S, Brown C and Halmi A. "Substance dependence and eating disorders: Impact of sequence on comorbidity" (1999). Comprehensive Psychiatry Vol 40. No5. Pp: 332-336.

(16) Sinha R \& O’Malley S.S. "Alcohol and eating disorders: implications for alcohol treatment and health services research". Alcohol Clin Exp Res, Vol 24, No 8. 2000. Pp: 1312-1319.

(17) Grilo CM, Levy KN, Becker DF, Edell WS \& Mc Glashan TH. Eating disorders in female inpatients with versus without substance use disorders. 1995. Addictive Behaviors. Vol 20. No 2. Pp: $255-260$. 
(18) Fernández Corres, B.; Lalaguna,B.; Rodríguez, C.\& González Pinto, A. "Comorbilidad de los trastornos de la conducta alimentaria y el abuso de sustancias". Trastornos adictivos. Vol 2 Num 1. Enero 2000.

(19) Bulik CM, Klump KL, Thornton L, Kaplan AS, Devlin B,... Fichter MM. “Alcohol use disorder comorbidity in eating disorders: a multicenter study". J Clin Psychiatry 2004. 65 (7): 1000-6.

(20) Brisman J. \& Siegel, M. "Bulimia and Alcoholism: Two Sides of the Same Coin?" Journal of Substance Abuse Treatment, Vol. 1, pp. 113-118.1984.

(21) Díaz Marsá, M. "Tratamiento de los trastornos de conducta alimentaria en sujetos con abuso de sustancias". Trastornos psiquiátricos y abuso de sustancias. Capítulo 20. Editorial Médica Panamericana. 2002.

(22) Franko DL, Dorer DJ, Keel PK, Jackson S, Manzo MP \& Herzog DB. Interactions between eating disorders and drug abuse. J Nerv Ment Dis 2008; 196:556-561.

(23) Holderness C.C. "Co-morbidity of eating disorders and substance abuse. Review of the literature". 1994. International Journal of Eating Disorders, Vol 16. No 1: 1-34.

(24) Dunne, F. J, Feeney S \& Schipperheijn J. "Eating disorders and alcohol misuse: features of an addiction spectrum". Postgrad Med J (1991) 67,112-113.

(25) Baker J H, Thornton L M, Strober M, Brandt H, Crawford S, Fichter M M et al. "Temporal sequence of comorbid alcohol use disorder and anorexia nervosa". Addict Behav 2013. 38 (3): 1704-9.

(26) Cooper, P.J; Taylor M.J; Cooper Z \& Fairburn C.G. (1987). The development and validation of the Body Shape Questionnaire. International Journal of Eating Disorders 6: 485-494.

(27) Zuckerman, M., Eysenck, S. B. y Eysenck, A. J. (1978). Sensation Seeking in England and American: crosscultural age and sex comparisons. Journal of Consulting and Clinical Psychology, 46, 139-149.

(28) Pérez, J. y Torrubia, R. (1986). Fiabilidad y validez de la versión española de la escala de búsqueda de sensaciones (forma V). Revista Latinoamericana de Psicología, 18, 7-22.

(29) Barratt, E. S. (1985) Impulsiveness defined within a systems model of personality. En E. P. Spielberger y J. N. Butcher (Eds.), Advances in Personality Assessment Hillsdale: Lawrence Erlbaum Associates, pp. 113-132.

(30) Luengo, M. A., Carrillo de la Peña, M. T. y Otero, J. M. (1991). The components of impulsiveness: a comparison of the I.7 Impulsiveness Questionnaire and the Barratt Impulsiveness Scale. Personality and Individual Differences, 12, 657-667.

(31) Spielberger, C. D., Gorsuch, R. L. y Lushene, R. E. (1970). STAI Manual for the StateTrait Anxiety Inventory(Self-Evaluation Questionnaire). Palo Alto California: Consulting Psychologists Press.

(32) Spielberger, C. D., Gorsuch, R. L. y Lushene, R. (1982). Manual del Cuestionario de Ansiedad Estado/Rasgo (STAI) (3 ${ }^{\text {a }}$ edic.). Madrid: TEA.

(33) Sanz, J. y Vázquez, C. (1998). Fiabilidad, validez y datos normativos del inventario para la depresión de Beck. Psicothema, 2, 303-318.

(34) Genis-Mendoza AD, Tovilla-Zarate CA y Nicolini H. "Avances en genómica de los trastornos de conducta alimentaria. 2013. Rev. Colomb. Psiquiatr Vol 42. Issue 4. Pp:350-355.

(35) Beresford TP \& Hall RC. "Food and drug abuse: the contrasts and comparisons of eating disorders and alcoholism”. Psychiatr Med 1989.7 (3):37-46. 
(36) Harrell Z.A.T, Slane J. D \& Klump K L. "Predictors of alcohol problems in college women: The role of depressive symptoms, disordered eating, and family history of alcoholism". Addictive Behaviors 34 (2009) 252-257.

(37) Franko DL; Dorer DJ; Keel PK; Jackson S; Manzo MP \& Herzog DB. 2005. How do eating disorders and alcohol use disorder influence each other? Int J Eat Disord 38: 3, pp: 200-207.

(38) Béhar R. "Consumo de alcohol y trastornos de la conducta alimentaria: evidencia, similitudes e implicancias. Rev. Chil. Neuro-psiquiatr. 2004 Vol 42(3): 183-194.

(39) Root T L, Pisetsky E.M, Thornton L, Lichtenstein P, Pedersen N L. \& Bulik C. M. "Patterns of Comorbidity of Eating Disorders and Substance Use in Swedish Females". Psychol Med. 2010; 40(1): 105-115. 